\title{
C-Reactive Protein-to-Albumin Ratio Predicts Sepsis and Prognosis in Patients with Severe Burn Injury
}

\author{
Yaohua Yu, ${ }^{1,2}$ Weiwei Wu, ${ }^{1,2}$ Yanyan Dong, ${ }^{3}$ and Jiliang $\mathrm{Li}^{1}{ }^{1,2}$ \\ ${ }^{1}$ Department of Plastic and Burn Surgery, Hwa Mei Hospital, University of Chinese Academy of Sciences, No. 41 Xibei Road, \\ Haishu District, Ningbo City, 315000 Zhejiang Province, China \\ ${ }^{2}$ Ningbo Institute of Life and Health Industry, University of Chinese Academy of Sciences, China \\ ${ }^{3}$ Ningbo College of Health Sciences, China
}

Correspondence should be addressed to Jiliang Li; lijiliang_nb@yeah.net

Received 8 December 2020; Revised 2 March 2021; Accepted 6 March 2021; Published 25 March 2021

Academic Editor: Helen C. Steel

Copyright (c) 2021 Yaohua Yu et al. This is an open access article distributed under the Creative Commons Attribution License, which permits unrestricted use, distribution, and reproduction in any medium, provided the original work is properly cited.

Background. Sepsis is a leading cause of mortality among severe burns. This study was conducted to investigate the predictive role of C-reactive protein-to-albumin ratio (CAR) for sepsis and prognosis in severe burns. Methods. Patients with severe burn injuries from 2013 to 2017 were enrolled and divided into septic and nonseptic groups based on the presence of sepsis within 30 days postburn. Independent risk factors for sepsis were performed by the univariate and multivariate logistic regression analyses. The association between CAR level at admission and postburn 30-day mortality was designed via the Kaplan-Meier method. Results. Of all the 196 enrolled patients, 83 patients developed sepsis within 30 days postburn injury, with an incidence of $42.3 \%$. TBSA percentage (OR: 1.65, 95\% CI: 1.17-2.32, $P=0.014$ ) and CAR at admission (OR: 2.25, 95\% CI: 1.33-3.56, $P=0.009$ ) were the two independent risk factors for sepsis in severe burns by the multivariate logistic regression analysis. A higher CAR level $(\geq 1.66)$ at admission was associated with a lower postburn 30 -day survival rate $(P=0.005)$. Conclusions. The CAR level at admission was an independent risk factor for sepsis and prognosis in severe burns.

\section{Introduction}

Globally, burn injury is one of the important causes of morbidity and mortality [1]. A severe burn trauma is a very common acute injury with the characteristics of aggressiveness [2]. Despite an advancing understanding of postburn resuscitation, organ support therapy, and wound treatment, patients with severe burn injuries are commonly accompanied with sepsis, septic shock, and organ dysfunction [3]. As reported in previous studies, sepsis is a frequent complication in patients with severe burn injury, which is closely associated with an increased incidence of morbidity and mortality [4]. Sepsis is a leading cause of mortality among severe burns, particularly when complicated by septic shock or multiple organ dysfunction syndromes (MODS). Due to the advancing understanding of pathophysiology and therapeutic strategies, the mortality rate has decreased significantly over the decades. However, the reported mortality in burn injury patients with sepsis is still as high as $20.3 \%$ [5].
As a result, early prediction for sepsis in severe burns is critically important for prognosis improvement. However, the diagnosis of sepsis in burn injuries is sometimes difficult due to the obscure diagnostic criteria and classical signs.

C-reactive protein (CRP), an acute-phase protein, is closely associated with systemic inflammatory status [6]. A recent study by Gulhan et al. [7] indicates CRP $(\geq 6 \mathrm{mg} / \mathrm{dL})$ as a risk factor for developing sepsis in pediatric patients with burn injuries. However, some others hold the view that CRP is a confounding factor in identifying sepsis in burn patients because the chronic inflammatory response is part of the normal stress response in patients with burn injuries [8]. A previous study has shown that the albumin (Alb) level may be a sensitive and specific biomarker for severity and a prognostic factor for mortality in burn patients [9]. CRP-to-Alb ratio (CAR), based on CRP and Alb, presents not only the inflammatory but also the nutritional status. With improved consistency when comparing with CRP or Alb alone, CAR is widely reported to be a prognostic factor in a variety of studies, 
including lung cancer [10], ovarian cancer [11], esophageal cancer [12], and critically ill patients admitted to the intensive care unit (ICU) [13]. However, to our knowledge, no studies have illustrated the role of CAR in sepsis and prognosis prediction in severe burns. We herein firstly investigated the prognostic values of CAR in severe burns.

\section{Material and Methods}

2.1. Patients. This was a retrospective study with the approval of the Medical Institutional Ethics Committee of our hospital (approval date: 2013.03.05, No. PJ-KY-NBEY-2013-002-08). Patients with severe burn injuries at the Department of Plastic and Burn Surgery, Hwa Mei Hospital, University of Chinese Academy of Sciences, from 2013 to 2017 were initially enrolled. The inclusion criteria were described as follows: (1) aged over 18 years, (2) with severe burn injury, (3) with signed informed consent, and (4) with a follow-up for at least 30 days. Exclusion criteria were as follows: (1) with the presence of sepsis upon admission; (2) with preexisting hepatic disease (i.e., liver cirrhosis and hepatitis), inflammatory diseases (i.e., osteoarthritis and inflammatory bowel disease), or other conditions (i.e., acute pancreatitis, acute infection, myocardial infarction, malignancies, malnutrition, and nephrosis) known to alter CRP or Alb; (3) combined with trauma or organ failure; and (4) incomplete data or refused to participate. As shown in the flow chart in Figure 1, 236 patients with severe burn injuries were initially enrolled and 40 were excluded according to the exclusion criteria. A total of 196 patients were enrolled in the final data analysis.

2.2. Treatment and Definitions. The treatment and management of enrolled patients were carried out following the relevant guidelines (including fluid resuscitation, nutritional support, surgery, management of inhalation injury, and prevention and treatment of infection). All the enrolled patients receive burn surgery (eschar excision and allogeneic skin covering for the first time, followed by repeatedly autologous "stamp" or mesh skin graft) within 30 days postburn. The fluid resuscitation was performed according to the protocol by the Third Military Medical University (TMMU) formula [14]. In summary, $1 \mathrm{~mL}$ of lactated Ringer's solution and $0.5 \mathrm{~mL}$ of plasma per $1 \%$ TBSA burn area per kilogram $(\mathrm{kg})$ were used for the first $24 \mathrm{~h} ; 0.5 \mathrm{~mL}$ of lactated Ringer's solution and $0.25 \mathrm{~mL}$ of plasma per 1\% TBSA burn area per kilogram $(\mathrm{kg})$ were used for the next $24 \mathrm{~h}$. In addition, $2 \mathrm{~L}$ water (as a $5 \%$ glucose solution) was additionally added as a daily basic requirement. The target of fluid resuscitation was set as hourly urine output $\geq 0.5 \mathrm{~mL} / \mathrm{kg} / \mathrm{h}$. Moreover, the hemodynamics targets by pulse indicator continuous cardiac output (PICCO) were set as cardiac index (CI) $>2.5 \mathrm{~L} / \mathrm{min} / \mathrm{m}^{2}$, intrathoracic blood volume index (ITBVI) $>600 \mathrm{~mL} / \mathrm{m}^{2}$, and lactic acid $<2 \mathrm{mmol} / \mathrm{L}$ [15].

Severe burn injury (including extremely severe burn) in this study was defined as a \% total burn surface area (TBSA ) $\geq 30 \%$ or third-degree TBSA $\geq 10 \%$. Sepsis was defined as life-threatening organ dysfunction which was caused by a dysregulated host response to infection according to the recommendations by the Third International Consensus Defini- tions for Sepsis and Septic Shock (Sepsis-3) [16]. Clinical criteria for sepsis were documented (or suspected) infection and an acute increase of $\geq 2$ Sequential Organ Failure Assessment (SOFA) points (a proxy for organ dysfunction) [16]. Sepsis within 30 days after burn injury was the primary observational endpoint, while mortality was set as the second endpoint.

2.3. Data Collection. The following data were collected: (1) demographics, including age, gender distribution, and body mass index (BMI); (2) preoperative comorbidities, including diabetes mellitus, hypertension, chronic coronary disease (CCD), and chronic obstructive pulmonary disease (COPD); (3) clinical data, including burn causes, admission time from burn, first excision time from burn, burn index, abbreviated burn severity index (ABSI) [17], Acute Physiology and Chronic Health Evaluation II (APACHE II) score and SOFA score [18] at admission, TBSA percentage, third-degree TBSA percentage, presence of inhalation injury, mechanical ventilation, and ICU admission; and (4) laboratory variables at admission, including hemoglobin ( $\mathrm{Hb})$, platelet (Plt), white blood cell (WBC), hematocrit (Hct), procalcitonin (PCT), blood urea nitrogen (BUN), creatinine, tumor necrosis factor- $\alpha$ (TNF- $\alpha$ ), CRP, and Alb. The burn index was calculated with the following formula: third-degree TBSA percentage $+1 / 2$ second-degree TBSA percentage [19].

2.4. Statistical Analysis. Data analysis was performed using SPSS 19.0 (SPSS, Inc., IA, USA) and GraphPad 8.0 (GraphPad Inc., CA, USA). Continuous variables with normal distribution are presented as mean \pm standard error (S.E.M.) and analyzed using the Student $t$-test. Continuous variables without normal distribution are presented as median with range and analyzed using the Mann-Whitney $U$ test. Categorical data are presented as a number with percentage $(n, \%)$ and compared using the chi-squared test or Fisher exact test. The predictive and cut-off values of variables for sepsis were established by receiving operating characteristic (ROC) curves. Risk factors associated with sepsis occurrence in severe burns were designed based on the univariate and multivariate binary logistic regression analyses using enter method. Multicollinearity test which includes variance inflation factor (VIF) was also conducted to evaluate the multicollinearity among factors. Only those factors with a $P$ value $<$ 0.05 in univariate logistic analysis were further included in the multivariate logistic regression model. The association between the CAR level at admission and postburn 30-day mortality was designed via the Kaplan-Meier method and $\log$-rank test. A $P$ value of $<0.05$ was considered statistically different.

\section{Results}

3.1. Patient Characteristics. A total of 196 patients with severe burn injuries were enrolled in this study. As shown in Figure 1, 83 patients developed sepsis within 30 days of postburn injury, with an incidence of $42.3 \%(83 / 196)$. The mean age of the cohort was 42.5 years and most patients were males $(62.2 \%, 122 / 196)$, with the main causes of thermal $(80.6 \%$, 


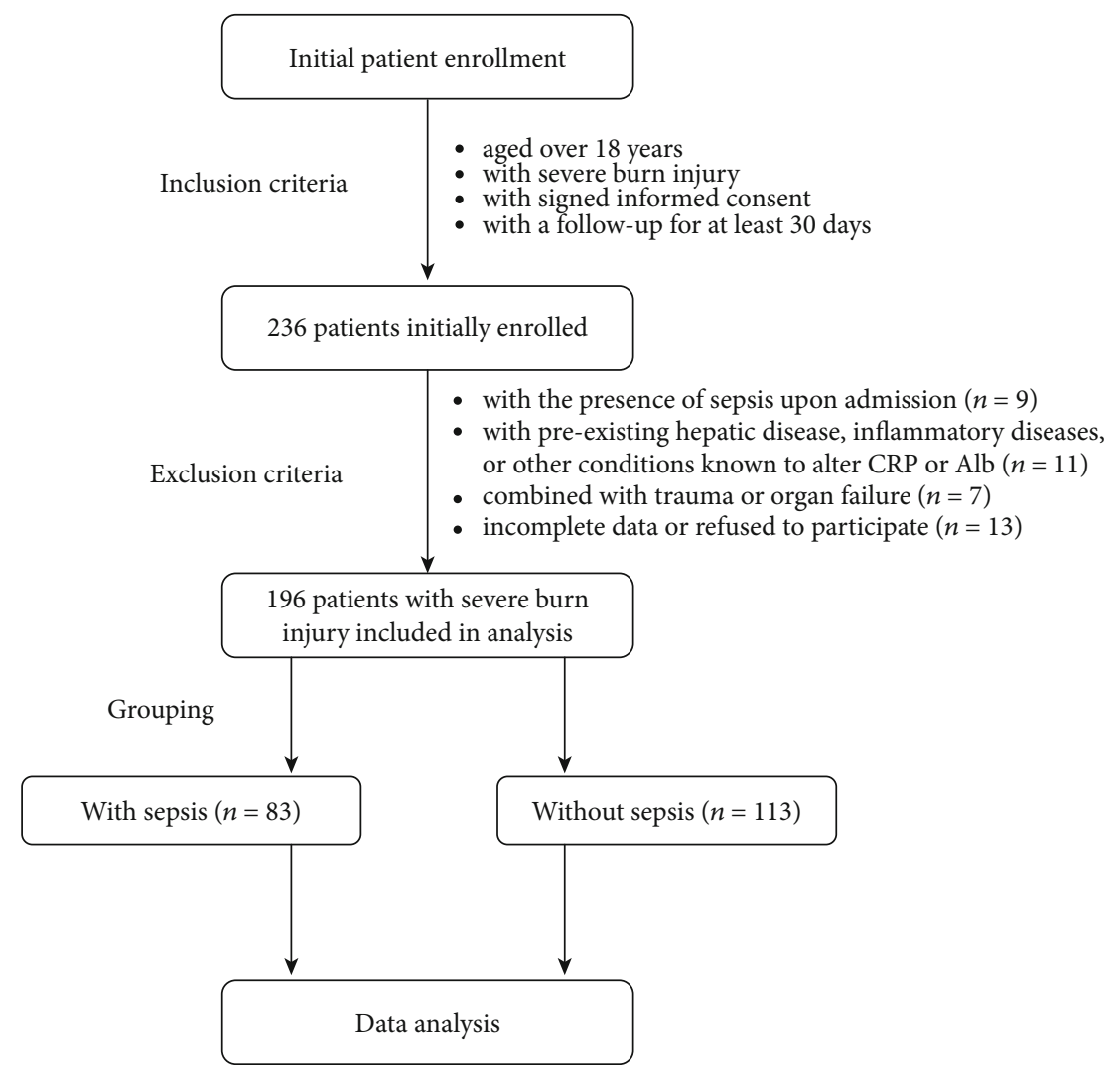

Figure 1: Flow chart. CRP: C-reactive protein; Alb: albumin.

$158 / 196)$. The majority of patients $(68.9 \%, 135 / 196)$ were admitted to ICU due to different reasons. Of the 135 patients admitted to ICU, 74 (54.8\%) were with shock and 81 (60.0\%) were with sepsis (see Table 1). The demographics and baseline characteristics were compared between patients with or without sepsis, which is presented in Table 2. Age, gender, BMI, causes, admission time from burn, preoperative comorbidities, APACHE II, and SOFA score at admission did not appear significantly different between sepsis and nonsepsis individuals $(P>0.05)$. The first excision time from burn $(P=0.037)$, burn index $(P=0.003)$, ABSI $(P=0.001)$, TBSA percentage $(P<0.001)$, and third-degree TBSA percentage $(P=0.006)$ were significantly higher in septic patients when comparing with nonseptic patients. Patients with the presence of inhalation injury were more susceptible to sepsis than those without inhalation injury $(P=0.021)$. Patients with sepsis were associated with higher rates of mechanical ventilation $(P=0.005)$ and ICU admission $(P<0.001)$, which was easily understandable. Table 3 demonstrates the comparative results of laboratory variables associated with sepsis in severe burns. Compared with nonsepsis patients, the serum expressions of TNF- $\alpha(P=0.012)$, PCT $(P<0.001)$, and CAR $(P<0.001)$ at admission were significantly higher in sepsis patients. The levels of $\mathrm{Hb}, \mathrm{Plt}, \mathrm{WBC}, \mathrm{Hct}$, creatinine, and BUN did not differ significantly between these two groups $(P>0.05)$.

3.2. Predictors for Sepsis. Of the potential risk factors $(P<0.05$ in Tables 2 and 3$)$, the predictive power of contin-
TABLE 1: Clinical and therapeutic parameters in severe burns admitted to the intensive care unit (ICU).

\begin{tabular}{lc}
\hline Parameters & $\begin{array}{c}\text { Severe burns admitted to ICU } \\
(n=135)\end{array}$ \\
\hline Shock, $n(\%)$ & $74(54.8)$ \\
Sepsis, $n(\%)$ & $81(60.0)$ \\
Septic shock, $n(\%)$ & $29(21.5)$ \\
Vasoactive drugs, $n(\%)$ & $90(66.7)$ \\
Blood transfusion & - \\
$\quad$ Red blood cell (u) & $7.4 \pm 1.6$ \\
$\quad$ Plasma (ml) & $4740 \pm 720$ \\
Platelet (therapeutic & $1.50 \pm 0.25$ \\
amount) & $0.14 \pm 0.02$ \\
Cryoprecipitate (u) &
\end{tabular}

uous variables for sepsis was evaluated by ROC curves and the results are displayed in Figure 2. The first excision time from burn was not a significant predictor $(P=0.716$, Figure 2(a)), while burn index $(P=0.0003$, Figure $2(\mathrm{~b}))$, ABSI $(P=0.013$, Figure $2(c))$, TBSA percentage $(P<0.0001$ , Figure $2(\mathrm{~d}))$, third-degree TBSA percentage $(P=0.0003$, Figure 2(e)), TNF- $\alpha(P=0.0007$, Figure 2(f)), and PCT $(P=0.016$, Figure $2(\mathrm{~g}))$ were all predictors for sepsis in severe burns. Among these factors, the CAR at admission was the most significant predictor with a cut-off value of 1.66 , an area 
TABLE 2: Clinical parameters associated with sepsis in severe burns.

\begin{tabular}{|c|c|c|c|}
\hline \multirow[b]{2}{*}{ Parameters } & \multicolumn{2}{|c|}{ Sepsis } & \multirow[b]{2}{*}{$P$ value } \\
\hline & $\begin{array}{c}\text { Yes } \\
(n=83)\end{array}$ & $\begin{array}{c}\text { No } \\
(n=113)\end{array}$ & \\
\hline Age (years) & $41.8 \pm 8.1$ & $43.1 \pm 7.5$ & 0.248 \\
\hline Gender, $n(\%)$ & - & - & 0.427 \\
\hline Male & $49(59.0)$ & $73(64.6)$ & - \\
\hline Female & $34(41.0)$ & $40(35.4)$ & - \\
\hline BMI $\left(\mathrm{kg} / \mathrm{m}^{2}\right)$ & $22.3 \pm 2.2$ & $22.2 \pm 2.5$ & 0.771 \\
\hline Causes & - & - & 0.908 \\
\hline Thermal & $68(81.9)$ & $90(79.6)$ & - \\
\hline Chemical & $10(12.0)$ & $16(14.2)$ & - \\
\hline Electronic & $5(6.0)$ & $7(6.2)$ & - \\
\hline Inhalation injury, $n(\%)$ & $30(36.1)$ & $24(21.2)$ & $0.021^{*}$ \\
\hline Admission time from burn $(\mathrm{h})$ & $18.2 \pm 3.9$ & $17.9 \pm 4.2$ & 0.611 \\
\hline Number of operations, $n(\%)$ & $4.3 \pm 0.4$ & $4.2 \pm 0.5$ & 0.125 \\
\hline $\begin{array}{l}\text { Mean duration of operation } \\
\text { (min) }\end{array}$ & $93.4 \pm 17.8$ & $90.3 \pm 20.1$ & 0.251 \\
\hline $\begin{array}{l}\text { First excision time from } \\
\text { burn }(\mathrm{d})\end{array}$ & $5.0 \pm 1.1$ & $4.7 \pm 0.9$ & $0.037^{*}$ \\
\hline Burn index & $41.4 \pm 6.3$ & $38.8 \pm 5.8$ & $0.003^{*}$ \\
\hline ABSI & $8.8 \pm 1.1$ & $8.3 \pm 1.0$ & $0.001^{*}$ \\
\hline APACHE II score at admission & $11.0 \pm 1.6$ & $10.7 \pm 1.2$ & 0.135 \\
\hline SOFA score at admission & $3.2 \pm 0.7$ & $3.1 \pm 0.6$ & 0.284 \\
\hline Mechanical ventilation, $n(\%)$ & $38(45.8)$ & $30(26.5)$ & $0.005^{*}$ \\
\hline ICU admission, $n(\%)$ & $81(97.6)$ & $54(47.8)$ & $<0.001^{*}$ \\
\hline $\begin{array}{l}\text { Preoperative comorbidities, } \\
n(\%)\end{array}$ & - & - & - \\
\hline Diabetes mellitus & $9(10.8)$ & $11(9.7)$ & 0.800 \\
\hline Hypertension & $14(16.9)$ & $17(15.0)$ & 0.730 \\
\hline CCD & $6(7.2)$ & $9(8.0)$ & 0.848 \\
\hline COPD & $5(6.0)$ & $7(6.2)$ & 0.961 \\
\hline TBSA percentage, $n(\%)$ & $47.3 \pm 8.1$ & $42.5 \pm 7.4$ & $<0.001^{*}$ \\
\hline $\begin{array}{l}\text { Third-degree TBSA } \\
\text { percentage, } n(\%)\end{array}$ & $15.9 \pm 3.7$ & $14.6 \pm 2.9$ & $0.006^{*}$ \\
\hline
\end{tabular}

BMI: body mass index; ABSI: abbreviated burn severity index; APACHE II: Acute Physiology and Chronic Health Evaluation II; SOFA: Sequential Organ Failure Assessment; ICU: intensive care unit; CCD: chronic coronary disease; COPD: chronic obstructive pulmonary disease; TBSA: total burn surface area. $P$ values were calculated by Student's $t$-test or chisquared test. ${ }^{*} P<0.05$.

under the curve (AUC) of 0.793 , a sensitivity of $74.34 \%$, and a specificity of $72.29 \%(P<0.0001$, Figure $2(\mathrm{~h}))$.

3.3. Risk Factors for Sepsis and Outcomes. The potential risk factors for sepsis in severe burns were investigated by the univariate and multivariate logistic regression analyses. No significant linearity was observed between sepsis and factors by multicollinearity test including VIF (Figure 3). As shown in Figure 3, six variables were potential risk factors by the univariate logistic regression analysis and were then placed into the multivariate model. TBSA percentage (OR: 1.65,
TABLE 3: Laboratory tests associated with sepsis in severe burns.

\begin{tabular}{lccc}
\hline $\begin{array}{l}\text { Laboratory tests } \\
\text { at admission }\end{array}$ & Yes $(n=83)$ & No $(n=113)$ & $P$ value \\
\hline $\mathrm{Hb}(\mathrm{g} / \mathrm{L})$ & $116.9 \pm 9.6$ & $118.9 \pm 8.8$ & 0.132 \\
$\mathrm{Plt}\left(\times 10^{9} / \mathrm{L}\right)$ & $282.1(82.0,662.6)$ & $269.8(88.0,722.3)$ & 0.543 \\
$\mathrm{WBC}\left(\times 10^{9} / \mathrm{L}\right)$ & $10.1 \pm 2.3$ & $9.7 \pm 1.9$ & 0.185 \\
$\mathrm{Hct}$ & $0.41 \pm 0.09$ & $0.42 \pm 0.08$ & 0.413 \\
$\mathrm{TNF}-\alpha(\mathrm{pg} / \mathrm{mL})$ & $358.4 \pm 32.3$ & $344.8 \pm 40.2$ & $0.012^{*}$ \\
$\mathrm{PCT}(\mu \mathrm{g} / \mathrm{L})$ & $2.3(0.05,130.3)$ & $1.2(0.05,114.0)$ & $<0.001^{*}$ \\
$\mathrm{Creatinine}$ & $88.0(66.0,134.0)$ & $79.0(62.0,112.0)$ & 0.324 \\
$(\mu \mathrm{mol} / \mathrm{L})$ & $7.7(4.5,9.3)$ & $6.9(4.8,8.9)$ & 0.278 \\
$\mathrm{BUN}(\mathrm{mmol} / \mathrm{L})$ & $55.3 \pm 20.1$ & $47.8 \pm 16.5$ & $0.005^{*}$ \\
$\mathrm{CRP}$ & $34.6 \pm 3.6$ & $35.9 \pm 4.5$ & 0.031 \\
$\mathrm{Alb}$ & $2.14(0.03,3.84)$ & $1.27(0.01,2.72)$ & $<0.001^{*}$ \\
$\mathrm{CAR}$ & &
\end{tabular}

Hb: hemoglobin; Plt: platelet; WBC: white blood cell; Hct: hematocrit; PCT: procalcitonin; BUN: blood urea nitrogen; TNF- $\alpha$ : tumor necrosis factor- $\alpha$; CAR: C-reactive protein-to-albumin ratio. $P$ values were calculated by Student's $t$-test or Mann-Whitney $U$ test. ${ }^{*} P<0.05$.

95\% CI: 1.17-2.32, $P=0.014$ ) and CAR at admission (OR: $2.25,95 \%$ CI: $1.33-3.56, P=0.009)$ were the two independent risk factors for sepsis in severe burns (see Figure 4). Of the 83 severe burns with sepsis, 26 died with a mortality rate of $31.3 \%$. The causes of mortality were septic shock $(n=11)$, acute respiratory distress syndrome (ARDS, $n=6$ ), intractable heart failure $(n=5)$, and others $(n=4)$.

3.4. CAR at Admission and Survival. A Kaplan-Meier survival plot was generated to evaluate the association between CAR at admission and postburn 30-day survival. As indicated in Figure 5, a higher CAR level $(\geq 1.66)$ at admission suggests a lower postburn 30-day survival rate (log-rank $P=0.005$ ).

\section{Discussion}

Currently, sepsis is newly defined as a type of life-threatening organ dysfunction, which results from a dysregulated host response to infection [20]. As described by previous reports, sepsis is a complicated immune response, with the characteristics of massive inflammatory mediators in the early stage followed by the rapidly developed immunocyte impairment and immunosuppression state [21]. Significantly elevated inflammatory cytokines due to the excessive inflammation in early sepsis often result in the development of organ injury and dysfunction, ultimately causing death [22]. Thus, early prediction of sepsis and relevant intervention are critically important for improving outcomes in severe burns. Age, inhalation injury, and burned percent of TBSA are widely reported as the cornerstones for predictive scoring models $[23,24]$ in patients with burns. Our results also indicated TBSA as an independent risk factor for sepsis in severe burns. A recent study by Sheckter et al. [25] identifies that the percentage of TBSA was significantly associated with adverse 
First excision time from burn

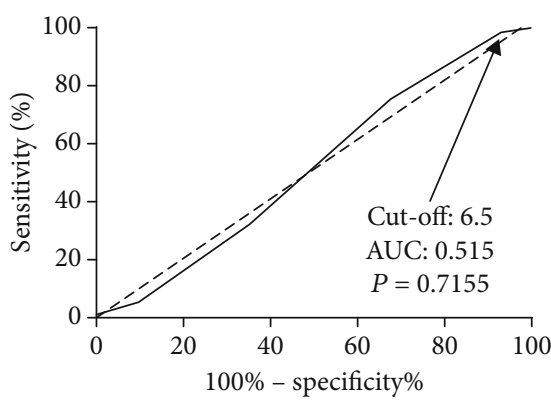

(a)

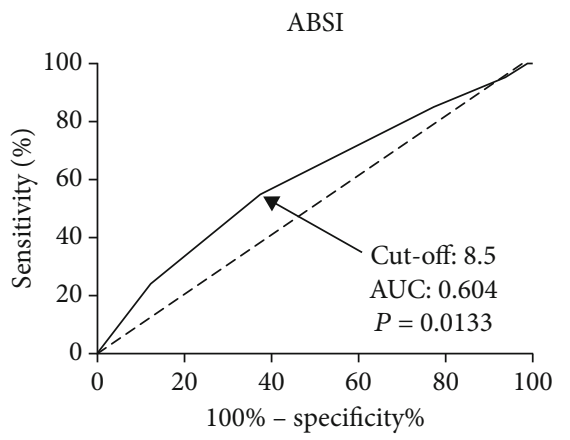

(c)

Third-degree TBSA percentage

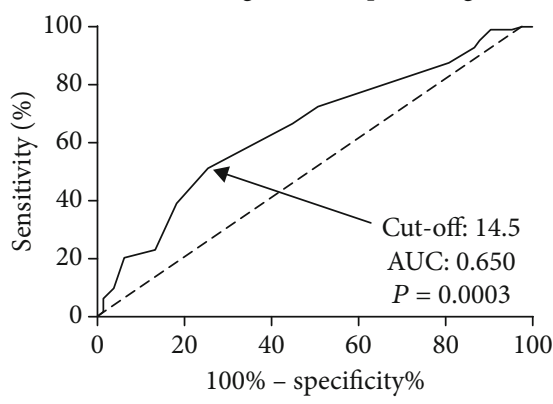

(e)

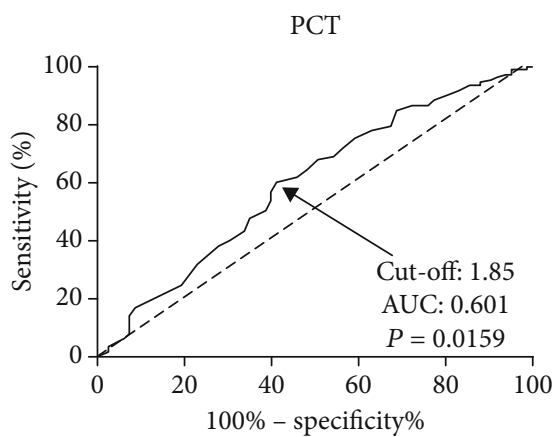

(g)

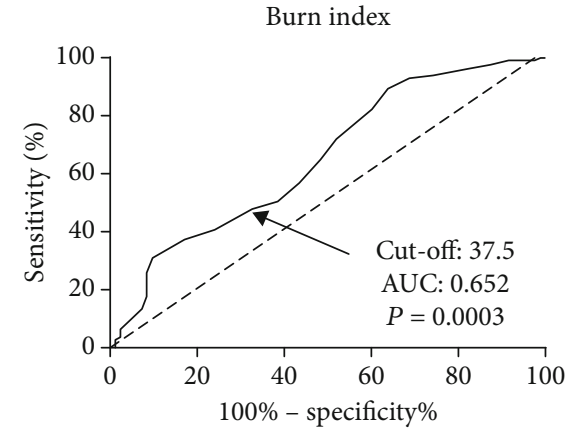

(b)

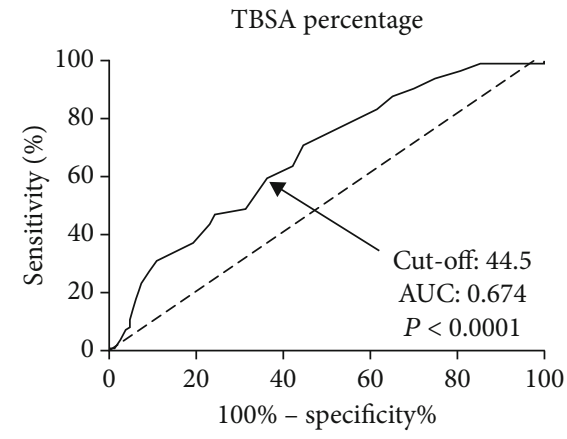

(d)

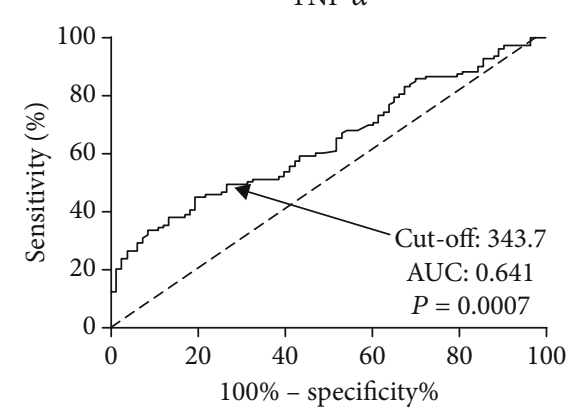

(f)

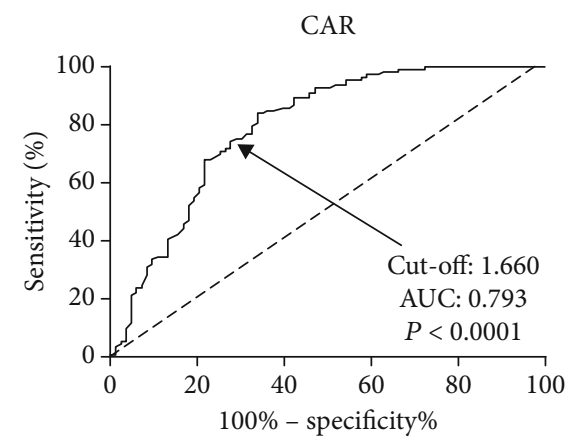

(h)

Figure 2: Predictive values of variables for sepsis in severe burns by ROC curve analysis. (a) First excision time from burn; (b) burn index; (c) ABSI; (d) TBSA percentage; (e) third-degree TBSA percentage; (f) TNF- $\alpha$; (g) PCT; (h) CAR. CAR at admission was the most significant predictor with a cut-off value of 1.66, an AUC of 0.793 , a sensitivity of $74.34 \%$, and a specificity of $72.29 \%(P<0.0001)$. CAR: C-reactive protein-to-albumin ratio; ABSI: abbreviated burn severity index; TBSA: total burn surface area; PCT: procalcitonin; TNF- $\alpha$ : tumor necrosis factor- $\alpha$; ROC: receiver operating characteristic; AUC: area under the curve. 


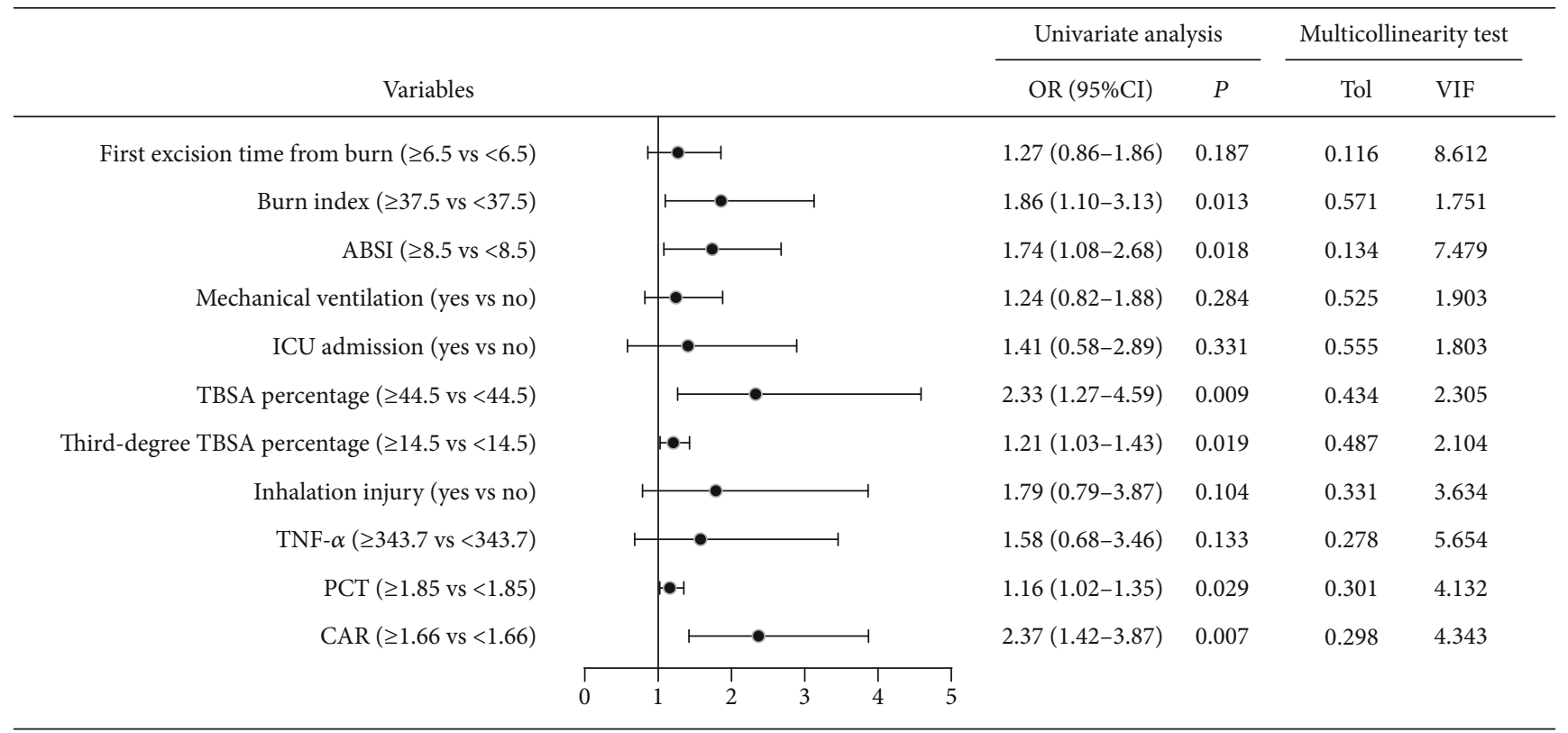

FIGURE 3: Forest plot of risk factors for sepsis by the univariate logistic regression analysis and multicollinearity test. ABSI: abbreviated burn severity index; TBSA: total burn surface area; ICU: intensive care unit; PCT: procalcitonin; TNF- $\alpha$ : tumor necrosis factor- $\alpha$; CAR: C-reactive protein-to-albumin ratio; OR: odds ratio; CI: confidence interval; Tol: tolerance; VIF: variance inflation factor.

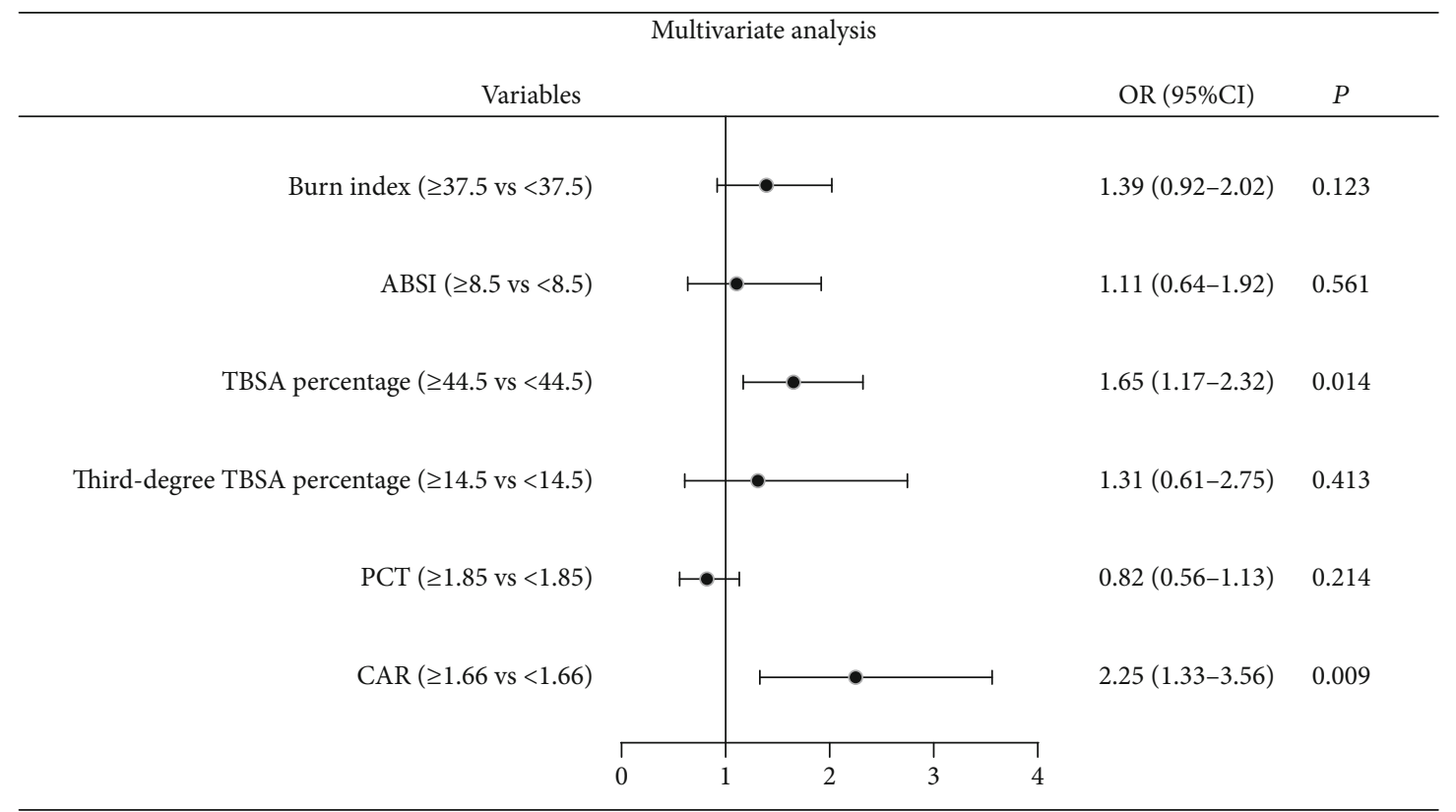

FIGURE 4: Forest plot of risk factors for sepsis by the multivariate logistic regression analysis. ABSI: abbreviated burn severity index; TBSA: total burn surface area; PCT: procalcitonin; CAR: C-reactive protein-to-albumin ratio; OR: odds ratio; CI: confidence interval.

outcomes in burn patients, which is quite in accordance with our results.

To our knowledge, this study firstly highlighted CAR at admission as an independent risk factor for sepsis and a novel prognostic factor among patients with severe burn injuries. Numerous studies have demonstrated a close correlation between CRP and sepsis. CRP is proposed to serve as a screening biomarker for neonatal sepsis [26]. A review in elderly geriatric patients by Ticinesi et al. [27] indicates that
CRP expression at admission is helpful for acute infection detection, particularly sepsis. Another study by Liu et al. [28] suggests that the combination of PCT and CRP is helpful for the early diagnosis of pneumonia and sepsis, as well as treatment response measurement and prognosis prediction in neonates. A meta-analysis by Tan et al. [29] also indicates the diagnostic role of CRP for sepsis in adult patients, although with lower accuracy and specificity when comparing with PCT. Besides, the correlation between Alb and sepsis 


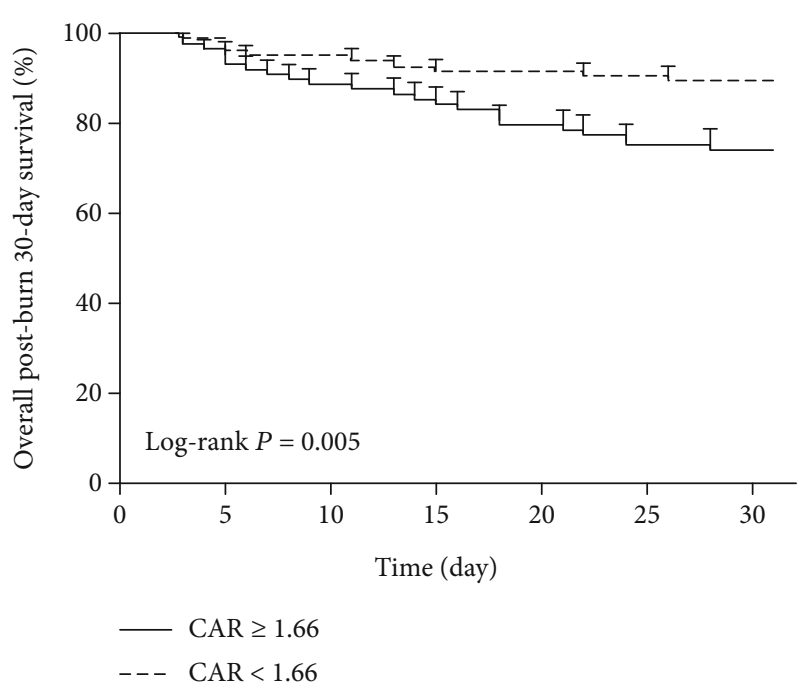

Figure 5: CAR at admission associated with postburn 30-day mortality by the Kaplan-Meier survival analysis. CAR: C-reactive protein-to-albumin ratio.

has also attracted a lot of attention. Reduced serum Alb concentrations are widely observed in patients with inflammatory states, including sepsis, due to the distribution alternation of Alb between extravascular and intravascular compartments induced by increased microvascular permeability [30]. Importantly, hypoalbuminemia correlates closely with increased mortality rates during hospitalization in intensive care units [31]. Besides the action as a plasma volume expander, Alb attracts more attention as the mediators of proinflammatory molecules and inflammation deserve more attention [32]. Alb is an important element in the moderation of the inflammatory response to bacterial infections by pathogen-associated molecular pattern- (PAMP-) albumin complexes via binding peptidoglycan, lipoteichoic acid, and lipopolysaccharide [33, 34]. Moreover, increased extravascular fluids induced by hypoalbuminemia also lead to some complications, such as healing abnormalities, edema, and increased susceptibility to sepsis [35]. In addition, excessive oxidative damage extensively exists in sepsis and often leads to cell dysfunction, death, and organ failure [36], while Alb acts as the main defense against this oxidative stress [37]. We hypothesized that CAR, which combines CRP and Alb, would imply the systemic inflammatory, nutritional, and immune statuses of patients. CAR could be much more sensitive, effective, and consistent than CRP or Alb alone. With the advantages, CAR has been widely recognized as a prognostic factor in various diseases, including metastatic renal cell carcinoma [38], ST elevation myocardial infarction [39], and hepatocellular carcinoma after curative resection [40]. CAR is reported to be an independent prognostic factor for 90-day mortality in septic individuals [41], which is in support of our conclusions. The close associations between CRP, Alb, and sepsis discussed above might be possible explanations for the predictive role of CAR for sepsis.

\section{Conclusions}

In conclusion, our results demonstrated that CAR at admission and TBSA percentage independently predicted sepsis in severe burns. A higher CAR $(\geq 1.66)$ at admission was associated with an increased postburn 30-day mortality.

\section{Data Availability}

Please contact the corresponding author (Jiliang Li, email: lijiliang_nb@yeah.net).

\section{Conflicts of Interest}

The authors declare that they have no conflicts of interest.

\section{Authors' Contributions}

YHY was responsible for project development, data collection, data analysis, and manuscript writing. WWW and YYD were responsible for data collection and project development. JLL was responsible for project development and data analysis.

\section{Acknowledgments}

The study was supported by the Medical Scientific Research Foundation of Zhejiang Province, China (No. 2021KY290) and Ningbo Key Discipline of Public Health (No. 2016020).

\section{References}

[1] V. H. Filaj and M. K. Belba, "Epidemiological trends of severe burns, 2009-2019: a study in the service of burns in Albania," Burns, 2020.

[2] A. Oryan, E. Alemzadeh, and A. Moshiri, "Burn wound healing: present concepts, treatment strategies and future directions," Journal of Wound Care, vol. 26, no. 1, pp. 5-19, 2017.

[3] R. C. Barber, C. C. Aragaki, F. A. Rivera-Chavez, G. F. Purdue, J. L. Hunt, and J. W. Horton, "TLR4 and TNF-alpha polymorphisms are associated with an increased risk for severe sepsis following burn injury," Journal of Medical Genetics, vol. 41, no. 11, pp. 808-813, 2004.

[4] J. Manning, "Sepsis in the burn patient," Critical Care Nursing Clinics of North America, vol. 30, no. 3, pp. 423-430, 2018.

[5] J. Yoon, D. Kym, J. Hur et al., "Comparative usefulness of sepsis-3, burn sepsis, and conventional sepsis criteria in patients with major burns," Critical Care Medicine, vol. 46, no. 7, pp. e656-e662, 2018.

[6] S. Eschborn and J. H. Weitkamp, "Procalcitonin versus Creactive protein: review of kinetics and performance for diagnosis of neonatal sepsis," Journal of Perinatology, vol. 39, no. 7, pp. 893-903, 2019.

[7] B. Gülhan, S. K. Yüksek, M. Hayran et al., "Infections in pediatric burn patients: an analysis of one hundred eighty-one patients," Surgical Infections, vol. 21, no. 4, pp. 357-362, 2020.

[8] D. G. Greenhalgh, J. R. Saffle, J. H. Holmes et al., "American Burn Association consensus conference to define sepsis and infection in burns," Journal of Burn Care \& Research, vol. 28, no. 6, pp. 776-790, 2007. 
[9] O. A. Aguayo-Becerra, C. Torres-Garibay, M. D. MacíasAmezcua et al., "Serum albumin level as a risk factor for mortality in burn patients," Clinics, vol. 68, no. 7, pp. 940-945, 2013.

[10] T. B. Deng, J. Zhang, Y. Z. Zhou, and W. M. Li, “The prognostic value of $C$-reactive protein to albumin ratio in patients with lung cancer," Medicine (Baltimore), vol. 97, no. 50, article e13505, 2018.

[11] Y. Liu, S. Chen, C. Zheng et al., "The prognostic value of the preoperative c-reactive protein/albumin ratio in ovarian cancer," BMC Cancer, vol. 17, no. 1, p. 285, 2017.

[12] Y. Wang, X. Hu, Y. Huang et al., "Prognostic value of the C-reactive protein to albumin ratio in esophageal cancer: a systematic review and meta-analysis," The Kaohsiung Journal of Medical Sciences, vol. 36, no. 1, pp. 54-61, 2019.

[13] T. K. Oh, I. A. Song, and J. H. Lee, "Clinical usefulness of Creactive protein to albumin ratio in predicting 30-day mortality in critically ill patients: a retrospective analysis," Scientific Reports, vol. 8, no. 1, p. 14977, 2018.

[14] G. Luo, Y. Peng, Z. Yuan et al., "Fluid resuscitation for major burn patients with the TMMU protocol," Burns, vol. 35, no. 8, pp. 1118-1123, 2009.

[15] M. Sánchez, A. García-de-Lorenzo, E. Herrero et al., “A protocol for resuscitation of severe burn patients guided by transpulmonary thermodilution and lactate levels: a 3-year prospective cohort study," Critical Care, vol. 17, no. 4, p. R176, 2013.

[16] M. Singer, C. S. Deutschman, C. W. Seymour et al., "The third international consensus definitions for sepsis and septic shock (sepsis-3)," Journal of the American Medical Association, vol. 315, no. 8, pp. 801-810, 2016.

[17] J. Tobiasen, J. M. Hiebert, and R. F. Edlich, "The abbreviated burn severity index," Annals of Emergency Medicine, vol. 11, no. 5, pp. 260-262, 1982.

[18] J. L. Vincent, A. de Mendonca, F. Cantraine et al., "Use of the SOFA score to assess the incidence of organ dysfunction/failure in intensive care units: results of a multicenter, prospective study. Working group on "sepsis-related problems" of the European Society of Intensive Care Medicine," Critical Care Medicine, vol. 26, no. 11, pp. 1793-1800, 1998.

[19] L. Qiu, C. Chen, S. J. Li et al., "Prognostic values of red blood cell distribution width, platelet count, and red cell distribution width-to-platelet ratio for severe burn injury," Scientific Reports, vol. 7, no. 1, p. 13720, 2017.

[20] A. Rhodes, L. E. Evans, W. Alhazzani et al., "Surviving sepsis campaign: international guidelines for management of sepsis and septic shock: 2016," Intensive Care Medicine, vol. 43, no. 3, pp. 304-377, 2017.

[21] R. S. Hotchkiss and I. E. Karl, "The pathophysiology and treatment of sepsis," The New England Journal of Medicine, vol. 348, no. 2, pp. 138-150, 2003.

[22] T. van der Poll, F. L. van de Veerdonk, B. P. Scicluna, and M. G. Netea, "The immunopathology of sepsis and potential therapeutic targets," Nature Reviews. Immunology, vol. 17, no. 7, pp. 407-420, 2017.

[23] J. S. Heng, O. Clancy, J. Atkins et al., "Revised Baux score and updated Charlson comorbidity index are independently associated with mortality in burns intensive care patients," Burns, vol. 41, no. 7, pp. 1420-1427, 2015.

[24] The Belgian Outcome in Burn Injury Study Group, “Development and validation of a model for prediction of mortality in patients with acute burn injury," The British Journal of Surgery, vol. 96, no. 1, pp. 111-117, 2009.

[25] C. C. Sheckter, C. Pham, D. Rochlin, Z. N. Maan, Y. Karanas, and C. Curtin, "The association of burn patient volume with patient safety indicators and mortality in the US," Burns, vol. 46, no. 1, pp. 44-51, 2020.

[26] F. Khan, "C-reactive protein as a screening biomarker in neonatal sepsis," Journal of the College of Physicians and Surgeons-Pakistan, vol. 29, no. 10, pp. 951-953, 2019.

[27] A. Ticinesi, F. Lauretani, A. Nouvenne et al., "C-reactive protein (CRP) measurement in geriatric patients hospitalized for acute infection," European Journal of Internal Medicine, vol. 37, pp. 7-12, 2017.

[28] G. B. Liu, X. Q. Cui, Z. B. Wang, L. Wen, and H. L. Duan, "Detection of serum procalcitonin and hypersensitive Creactive protein in patients with pneumonia and sepsis," Journal of Biological Regulators and Homeostatic Agents, vol. 32, no. 5, pp. 1165-1169, 2018.

[29] M. Tan, Y. Lu, H. Jiang, and L. Zhang, "The diagnostic accuracy of procalcitonin and C-reactive protein for sepsis: a systematic review and meta-analysis," Journal of Cellular Biochemistry, vol. 120, no. 4, pp. 5852-5859, 2019.

[30] A. Fleck, G. Raines, F. Hawker et al., "Increased vascular permeability: a major cause of hypoalbuminaemia in disease and injury," Lancet, vol. 1, no. 8432, pp. 781-784, 1985.

[31] SAFE Study Investigators, "Effect of baseline serum albumin concentration on outcome of resuscitation with albumin or saline in patients in intensive care units: analysis of data from the saline versus albumin fluid evaluation (SAFE) study," BMJ, vol. 333, no. 7577, p. 1044, 2006.

[32] A. Artigas, J. Wernerman, V. Arroyo, J. L. Vincent, and M. Levy, "Role of albumin in diseases associated with severe systemic inflammation: pathophysiologic and clinical evidence in sepsis and in decompensated cirrhosis," Journal of Critical Care, vol. 33, pp. 62-70, 2016.

[33] T. L. Gioannini, D. Zhang, A. Teghanemt, and J. P. Weiss, “An essential role for albumin in the interaction of endotoxin with lipopolysaccharide-binding protein and sCD14 and resultant cell activation," The Journal of Biological Chemistry, vol. 277, no. 49, pp. 47818-47825, 2002.

[34] G. A. Esparza, A. Teghanemt, D. Zhang, T. L. Gioannini, and J. P. Weiss, "Endotoxin-albumin complexes transfer endotoxin monomers to MD-2 resulting in activation of TLR4," Innate Immunity, vol. 18, no. 3, pp. 478-491, 2011.

[35] M. Lehnhardt, H. J. Jafari, D. Druecke et al., "A qualitative and quantitative analysis of protein loss in human burn wounds," Burns, vol. 31, no. 2, pp. 159-167, 2005.

[36] M. Singer, "The role of mitochondrial dysfunction in sepsisinduced multi-organ failure," Virulence, vol. 5, no. 1, pp. 6672, 2013.

[37] M. Taverna, A. L. Marie, J. P. Mira, and B. Guidet, "Specific antioxidant properties of human serum albumin," Annals of Intensive Care, vol. 3, no. 1, p. 4, 2013.

[38] S. Konishi, S. Hatakeyama, T. Tanaka et al., "C-reactive protei$\mathrm{n} /$ albumin ratio is a predictive factor for prognosis in patients with metastatic renal cell carcinoma," International Journal of Urology, vol. 26, no. 10, pp. 992-998, 2019.

[39] T. Çınar, M. Çağdaş, İ. Rencüzoğulları et al., "Prognostic efficacy of C-reactive protein/albumin ratio in ST elevation myocardial infarction," Scandinavian Cardiovascular Journal, vol. 53, no. 2, pp. 83-90, 2019. 
[40] Y. Ren, X. Fan, G. Chen, D. Zhou, H. Lin, and X. Cai, "Relacion proteina $\mathrm{C}$ reactiva/albumina preoperatoria para predecir la mortalidad y la recurrencia de los pacientes despues de la reseccion curativa de carcinoma hepatocelular," Medicina Clínica (Barcelona), vol. 153, no. 5, pp. 183-190, 2019.

[41] O. T. Ranzani, F. G. Zampieri, D. N. Forte, L. C. P. Azevedo, and M. Park, "C-reactive protein/albumin ratio predicts 90 day mortality of septic patients," PLoS One, vol. 8, no. 3, article e59321, 2013. 